\title{
Light Harvesting and Carrier Transport in Core/Barrier/Shell Semiconductor Nanocrystals
}

\author{
Eva A. Dias, Samuel L. Sewall, and Patanjali Kambhampati*
}

\section{Supporting Information.}

\section{Materials and methods.}

CdSe core nanocrystals were prepared from $\mathrm{CdO}$ and Se using the alternate precursor method developed by Peng and co-workers. ${ }^{1}$ The only modification was the substitution of hexadecylamine (HDA) for octadecylamine (ODA). The CdSe nanocrystals were capped TOPO and HDA. The layered $\mathrm{CdSe} / \mathrm{ZnS} / \mathrm{CdSe}$ systems were prepared using the modified successive ionic layer adsorption and reaction (SILAR) method developed by Peng and co-workers. ${ }^{1-3}$ This method involves the addition of precursor solutions for each of the ions involved to a reaction flask containing a known amount and size of CdSe nanocrystals. Just enough of each precursor was added to grow one monolayer of $\mathrm{ZnS}$ or $\mathrm{CdSe}$. The precursor solutions for $\mathrm{Cd}$ and $\mathrm{Zn}$ were prepared by heating $\mathrm{CdO}$ and $\mathrm{ZnO}$ in oleic acid $(\mathrm{OA})$ and 1 -octadecene (ODE) to $250^{\circ} \mathrm{C}$ and $300^{\circ} \mathrm{C}$, respectively, under argon atmosphere until the solution became clear. The $\mathrm{Cd}$ and $\mathrm{Zn}$ precursor solutions were allowed to cool to and were kept at $70^{\circ} \mathrm{C}$. The S precursor was prepared by heating $\mathrm{S}$ in $\mathrm{ODE}$ to $100^{\circ} \mathrm{C}$ under argon to dissolve. The Se precursor was prepared by dissolving Se powder in tributylphosphine (TBP) and ODE.

The reaction solution of CdSe cores, typically $2.8 \times 10^{-8}$ mol, in ODA and ODE was heated under argon to $200^{\circ} \mathrm{C}$, at which temperature the $\mathrm{Zn}$ precursor was added followed 5 min later by the addition of the $\mathrm{S}$ precursor. The layer was allowed to react for $25 \mathrm{~min}$ before another layer was added. The temperature was reduced to $190^{\circ} \mathrm{C}$ for the addition of the CdSe layers 
which were added in a similar way to the $\mathrm{ZnS}$ layers. For this study 3 monolayers (ML) of $\mathrm{ZnS}$ were added, followed by $3 \mathrm{ML}$ of CdSe. For these materials $3 \mathrm{ML}=1 \mathrm{~nm}$.

For some spectra, the ligands on the CdSe cores and $\mathrm{CdSe} / \mathrm{ZnS} / \mathrm{CdSe}$ core/barrier/shells were first exchanged for pyridine. ${ }^{4}$ For the ligand exchange, the nanocrystals were dissolved in a small amount of neat pyridine and heated at $50^{\circ} \mathrm{C}$ for $1 \mathrm{hr}$. The nanocrystals were precipitated out of solution using hexanes/acetone (6:1) and dissolved in methanol or pyridine.

Pyridine, toluene, methanol, hexanes, and acetone were obtained from Fisher. All other chemicals were purchased from Aldrich. The steady state absorption measurements were performed on a Varian Cary 300 UV/Visible spectrophotometer. The photoluminescence (PL) and photoluminescence excitation (PLE) spectra were measured on a Spex Fluromax-2 spectrofluorometer. For these measurements the nanocrystals were dissolved in toluene, with the exception of the pyridine coated particles which were dissolved in methanol or pyridine. Emission measurements were taken with an OD $\sim 0.04$ for emission from the core, and $\sim 0.01$ for emission from the shell. The synthesis and characterization was performed four times to verify reproducibility of the results.

\section{Quantum yield spectra.}

The quantum yield spectra are proportional to the PLE spectra divided by the absorption spectra. Emission was monitored at $2.0 \mathrm{eV}$ (core) and $2.3 \mathrm{eV}$ (shell). Overlap between the two phases was $<1 \%$. In the case of the core/barrier/shell materials, subtraction methods were used to identify the absorption from each CdSe phase. The contribution from the core phase was taken as identical to the absorption spectrum of the bare core. This was motivated by the observation that the emission spectrum from the core phase and the band edge absorption spectra are nearly 
identical for the bare core and the core/barrier/shell material. By dividing the PLE for the core/shell (core) by the absorption from the core, we obtain the quantum yield spectrum for the core/shell (core). This nomenclature refers to emission from the core phase of the core/barrier/shell material. In the case of the shell, the absorption was taken as the difference between the core/barrier/shell and the bare core. The band edge absorption of ZnS nanocrystals is higher than the energies probed here. By dividing the PLE for the core/shell (shell) by the absorption from the shell phase, we obtain the quantum yield spectrum for the core/shell (shell).

\section{Branching ratio for shell emission.}

Photons absorbed by the shell may emit from the shell or from the core. Table I quantifies the extent to which the shell emits from each phase. The shell has $6.5 \mathrm{x}$ as much absorbance as the core, at $3.1 \mathrm{eV}$. This ratio is close to the volume ratio of 7:1. The integrated area of the PL is shown in Table I for the shell relative to the core. The core is $5 \mathrm{x}$ brighter than the shell even though it has $\sim 7 \mathrm{x}$ less absorbance. The PLE values are based upon normalization to the band edge of the core, where the shell has no influence. Scaling in this manner allows separation of the core and shell contributions to emission from the core phase. For the bare core, the PLE is $1.5 \mathrm{x}$ greater at $3.1 \mathrm{eV}$ than for band edge excitation. For the core/shell (core) the PLE is $2.5 \mathrm{x}$ greater which reflects the influence of the shell. Thus the shell contribution to emission from the core is $40 \%$ and the core contribution is $60 \%$ for the core/barrier/shell material. From the PLE, we see that the core/barrier/shell material is $1.7 \mathrm{x}$ brighter at $3.1 \mathrm{eV}$ than a bare core. Since the shell can emit in its own phase or induce emission from the core, it is interesting to compute the branching ratio for emission. For normalized emission from the core, a fraction of 0.6 came originated from the core while a fraction of 0.4 originated from the shell. The shell emission is 
0.2 relative to the total core phase emission. Therefore the shell is twice as likely to induce emission from the core as it is to emit from its own phase. 
Table I. Spectral amplitudes for each phase at an excitation energy of $3.1 \mathrm{eV}$.

\section{Core/barrier/shell Core Shell}

\begin{tabular}{llll}
\hline Absorbance $^{\mathbf{a}}$ & 14.9 & 1.9 & 13.0 \\
PL (relative area) & & 1 & 0.2 \\
PLE $^{\mathbf{c}}$ (fractional) & 2.5 & 1.5 & 1.0 \\
$\begin{array}{l}\text { Brightness relative } \\
\text { to core }\end{array}$ & $1.7 \mathrm{x}$ & & \\
$\begin{array}{l}\text { Transfer from shell } \\
\text { vs. emission from } \\
\text { shell }\end{array}$ & $2: 1$ & &
\end{tabular}

${ }^{a}$ Absorbance is normalized to the band edge of the core, where only the core absorbs. Shell absorbance comes from subtraction of core from core/barrier/shell.

${ }^{\mathrm{b}} \mathrm{PL}$ is for excitation at $3.1 \mathrm{eV}$. The integrated area of the shell is relative to that of the core.

${ }^{\mathrm{c}} \mathrm{PLE}$ is for emission from the core phase and is normalized to the band edge of the core, where only the core absorbs. The shell contribution to emission from the core comes from subtraction of core from core/barrier/shell.

${ }^{\mathrm{d}}$ Excitons in the shell can either cause emission from the core or from the shell. $40 \%$ of the core emission came from the shell (c). This $40 \%$ of the core emission is $2 \mathrm{x}$ the area of the shell emission.

\section{References.}

(1) J. J. Li, Y. A. Wang, W. Guo, J. C. Keay, T. D. Mishima, M. B. Johnson, and X. Peng, J. Am. Chem. Soc. 125, 12567 (2003).

(2) D. Battaglia, J. Li Jack, Y. Wang, and X. Peng, Angew. Chem., Int. Ed. Engl. 42, 5035 (2003).

(3) D. Battaglia, B. Blackman, and X. Peng, J. Am. Chem. Soc. 127, 10889 (2005).

(4) M. Kuno, J. K. Lee, B. O. Dabbousi, F. V. Mikulec, and M. G. Bawendi, J. Chem. Phys. 106, 9869 (1997). 